\title{
Following FRET through Five Energy Transfer Steps: \\ Spectroscopic Photobleaching, Recovery of Spectra, and a Sequential Mechanism of FRET
}

\author{
Toni S. Forde ${ }^{\dagger}$ and Quentin S. Hanley ${ }^{\dagger *}$ \\ †Department of Biological and Chemical Sciences \\ University of the West Indies \\ Cave Hill Campus \\ St. Michael, Barbados \\ f School of Biomedical and Natural Sciences \\ Nottingham Trent University \\ Clifton Lane \\ Nottingham NG11 8NS \\ United Kingdom
}

* Corresponding author: Q. S. Hanley

E-mail: qhanley@uwichill.edu.bb, quentin.hanley@ntu.ac.uk. 


\begin{abstract}
:
We report the acquisition and analysis of spectrally resolved photobleaching data from a model system designed to exhibit FRET. Spectrally resolved photobleaching can be used to determine the presence of FRET in these systems and to investigate multi-step mechanisms of energy transfer. The model system was a previously described set of fluorescent beads consisting of a system of 6 fluorophores. In standard photobleaching experiments to determine FRET, bleaching of an acceptor molecule resulting in recovery of donor intensity or changes in photobleaching kinetics are used as indicators of FRET. Here, we use the Bateman equations to model growth and decay in a photobleaching experiment. Linked donor-acceptor growth and decay is used as an indicator of FRET. The apparatus required is relatively simple when compared to lifetime imaging systems. Several data analysis strategies, rigorous model building, global fitting procedures, and error analysis are presented. Using these procedures a five-step sequential mechanism of energy transfer was selected for these beads.
\end{abstract}

Key Words: Photobleaching, multi-fluorophore FRET, pbFRET, Model Building. 


\section{Introduction}

The phenomenon of FRET has been used for many years as a spectroscopic ruler for the study of molecular interactions in both in vivo and in vitro systems. ${ }^{1-8}$ The recent advances in developing new fluorescent probes, instrumentation and methodologies have greatly increased the utility and scope of $\mathrm{FRET}^{9}$ and an overview of methods for FRET analysis recently appeared ${ }^{10}$. While much recent work on FRET has involved various lifetime imaging approaches and ratiometric techniques, one of the simplest methods involves the observation of photobleaching kinetics.

Photobleaching is a dynamic process in which a chromophore undergoes photoinduced chemical destruction upon exposure to excitation light. This process has been used to study complex mixtures of absorbing species. ${ }^{11-13}$ In fluorophores, the bleaching process results in molecules losing their ability to fluoresce. ${ }^{14-16}$ Photobleaching is often involved in fluorescence measurements of biological specimens and is detected as a reduction in fluorescence emission over the illumination period. ${ }^{17}$ This phenomenon is often considered an undesirable side effect in fluorescence measurements because it decreases the signal and lowers the signal-to-background ratio $^{18}$. Notwithstanding this, photobleaching has been utilized in a number of fluorescent measurement techniques. A few examples of techniques that take advantage of the photobleaching phenomenon include polarized photobleaching, fluorescence loss in photobleaching (FLIP), ${ }^{19,20}$ fluorescence recovery after photobleaching (FRAP) ${ }^{19}, 21$, and photobleaching fluorescence resonance energy transfer (pbFRET). The pbFRET method is a quantitative approach with the added value of not requiring sophisticated and expensive instrumentation. $^{22}$ In this technique, FRET is quantified by measuring the photobleaching kinetics of either the donor or acceptor in a FRET pair. In donor pbFRET, ${ }^{23,24}$ the energy 
transfer process reduces the number of excited-state donors available for photodestruction; thus slowing the decay rate of photobleaching. If photobleaching of the acceptor fluorophore occurs, the result is an increase in donor intensity since it is no longer quenched by the FRET process.

The competitive nature of FRET and photobleaching is often exploited to allow for the indirect measurement of FRET via its effects on donor photobleaching. ${ }^{25}$ It has been previously demonstrated that the efficiency of energy transfer between the donor-acceptor pair can be evaluated from photobleaching data. ${ }^{22,23,25,26}$ Photobleaching techniques have also been used to reveal the dynamics underlying the steady-state distribution of a fluorophore ${ }^{20}$ and the dynamics of single light-harvesting complexes. ${ }^{27}$

The analysis of photobleaching kinetics in fluorescence spectroscopy has been the focus of a number of past studies. Benson et $a l^{28}$ measured the photobleaching kinetics of various fluorophores in living and fixed cells and demonstrated that the photobleaching process followed first-order reaction kinetics with spatially heterogeneous and varied rate constants. Experimental studies have shown that photobleaching data does not always follow this single-exponential behavior. Szabo et al. ${ }^{26}$ in their investigation of epitope mapping by FRET reported that the photobleaching kinetics of their test system, a FITC (fluorescein isothiocyanate) moiety attached to an immunoglobulin, followed a double-exponential process. Song et al. ${ }^{14,16}$ investigated the photobleaching of fluorescein through a systematic analysis of photobleaching mechanisms and observed that photobleaching of fluorescein in microscopy is generally not a single-exponential process. Young et $\mathrm{al}^{23}$ used least squares methods to fit experimental photobleaching data to a multi-exponential function of the form: 


$$
F_{t}=F_{0} \sum \alpha_{i} e^{-k_{i} t}
$$

where $F_{0}$ is the initial fluorescence, $F_{t}$ is the fluorescence at time $t, \alpha_{i}$ are the fractional amplitudes of the various decay rates, and $k_{i}$ are the various apparent photobleaching rates. Error estimates for the fitted parameters were approximated using linear estimates. The results of the data analysis clearly showed that three exponentials were required to give a satisfactory fit to their experimental data. Gadella and Jovin ${ }^{29}$ illustrated the utility of pbFRET as a method for the quantitative evaluation of spatially resolved FRET and for generating contrast by allocating specific cellular structures to a particular decay component in a fluorescence image.

While multi-exponential fits to photobleaching data are known, previous studies have been restricted to a limited number of fluorophores, donor photobleaching of single donor-acceptor pairs, or intensity recovery after acceptor photobleaching. Here an analysis of photobleaching kinetics in a multi-fluorophore system is presented with a description of statistical model building procedures. The analysis involves the fitting of exponential models of growth and decay to selected wavelengths. The model system consists of a previously described set of fluorescent beads $^{30}$ containing multiple fluorophores. In this study, the appearance of growth in a photobleaching model which is equal to the rate of acceptor decay, is utilized as an indicator of FRET. The advantage of this approach is that it employs a series of simple kinetic models and uses statistical tests to justify the inclusion of additional parameters or to select or reject a particular model. The apparatus required to obtain the initial data sets is relatively simple when 
compared to lifetime imaging systems and allows "mechanisms" of energy transfer in multifluorophore systems to be tested.

\section{Theory Section}

Kinetic Models. Young et al. ${ }^{23}$ have treated photobleaching of a donor in a donor-acceptor pair as a first order reaction from the excited state.

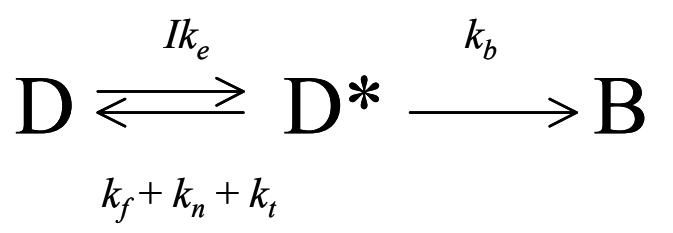

In this model, $\mathrm{D}$ is a donor molecule which can be excited to a state, $\mathrm{D}^{*}$, at a rate determined by the product of intensity, $I$, and the intensity dependent rate constant for excitation, $k_{e}$. The first order de-excitation rate processes (fluorescence emission, non-radiative deactivation, and energy transfer, designated $k_{f}, k_{n}$, and $k_{t}$, respectively) compete with photobleaching $\left(k_{b}\right)$ for the excited state.

This is not a full treatment as it does not include growth of donor by bleaching of the acceptor, however, a complete scheme is somewhat complex (figure 1). While the reaction scheme depicted in figure 1 is complex, it is important as a basis from which to apply simplifying assumptions. First, under conditions of constant illumination intensity, the product of the intensity and any excitation rate constant can be treated as a constant. Second, the illumination intensity is considered to be sufficiently low allowing all processes involving $\mathrm{D}^{*} \mathrm{~A}^{*}$ to be 
neglected. Third, a special case can be considered if the donor and acceptor are sufficiently close together that $k_{F R E T} \gg>>\left(k_{r d}+k_{n d}\right)$. This implies that the donor does not bleach in the presence of the acceptor and only bleaches after acceptor bleaching. Applying these three assumptions leaves a situation very similar to radioactive series decay and simplifies the treatment of multiple fluorophores. Combining rate constants and gathering terms yields:

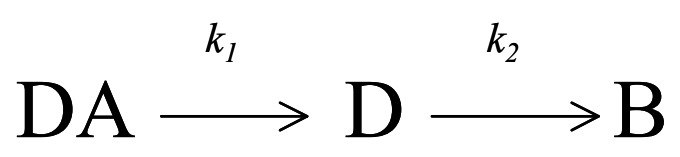

3.

In equation $3, k_{1}$ is the rate of acceptor bleaching in the donor acceptor complex and $k_{2}$ is the rate of free donor bleaching. This simplified model has several consequences. First, if DA and D are linked in such a series the growth of $\mathrm{D}$ has to proceed at the same rate as the destruction of DA. Second, the rate constants for any species would be expected to be wavelength invariant. Third, free donor or other species not participating in FRET can be treated as parallel processes.

The following shorthand notation will be used: for a series of energy transfer steps among interacting fluorophores exhibiting efficient energy transfer an arrow represents energy transfer between a donor and an acceptor (e.g. $A_{3} \rightarrow A_{2} \rightarrow A_{1}$ ). Parallel processes not involving energy transfer will be indicated with a plus sign (e.g. $A_{3}+A_{2}+A_{1}$ ) and mixed processes will combine the two (e.g.: $\left.\left(A_{3} \rightarrow A_{2} \rightarrow A_{1}\right)+A_{3}+A_{2}+A_{1}\right)$. The latter case represents a ternary mix in which a portion of each species participates in energy transfer and the remainder does not. 
Processes of the type in scheme 3 are described by the Bateman equations, which were derived by Bateman in 1910 as generalizations of the exponential laws governing the growth and decay of radioactive materials. ${ }^{31}$ These equations are solutions to the first order differential equations: ${ }^{\dagger}$

$$
\begin{aligned}
& \frac{d A_{1}}{d t}=-k_{1} A_{1} \\
& \frac{d A_{2}}{d t}=k_{1} A_{1}-k_{2} A_{2} \\
& \frac{d A_{n}}{d t}=k_{n-1} A_{n-1}-k_{n} A_{n}
\end{aligned}
$$

Equations 4-6 have solutions of the form:

$$
\begin{aligned}
& A_{1}(t)=A_{1} e^{-k_{1} t} \\
& A_{2}(t)=A_{2}\left(h_{12} e^{-k_{1} t}+h_{22} e^{-k_{2} t}\right) \\
& A_{n}(t)=A_{n}\left(h_{1 n} e^{-k_{1} t}+h_{2 n} e^{-k_{2} t}+\ldots+h_{n n} e^{-k_{n} t}\right)
\end{aligned}
$$

where $A_{1}, \ldots, A_{n}$ are constants related to the amount of the parent and daughter species present. In radioactive decay, it would be assumed that $A_{1}, \ldots, A_{n}$ are equal to the amount of species $A_{1}$ at

${ }^{\dagger}$ In the context of radioactive decay, the first order rate constants, $k_{1}, k_{2}, \ldots, k_{n}$ are usually written $\lambda_{1}, \lambda_{2}, \ldots, \lambda_{n}$. 
time zero, a situation which does not apply in this context. The quantities, $h_{12}, h_{22}, h_{1 n}$, etc, depend on the first order rate constants as shown in equations 10 and 11 .

$$
\begin{aligned}
& h_{12}=\frac{k_{1}}{k_{2}-k_{1}}, h_{22}=-\frac{k_{1}}{k_{2}-k_{1}} \\
& h_{1 n}=\frac{k_{1}}{k_{n}-k_{1}} \cdot \frac{k_{2}}{k_{2}-k_{1}} \cdots \frac{k_{n-1}}{k_{n-1}-k_{1}}, h_{2 n}=\frac{k_{1}}{k_{1}-k_{2}} \cdot \frac{k_{2}}{k_{n}-k_{2}} \cdots \frac{k_{n-1}}{k_{n-1}-k_{2}}, \\
& h_{n n}=\frac{k_{1}}{k_{1}-k_{n}} \cdot \frac{k_{2}}{k_{2}-k_{n}} \cdots \frac{k_{n-1}}{k_{n-1}-k_{n}}
\end{aligned}
$$

While these equations are of some complexity, it should be noted that only two additional parameters are needed for each additional species observed at a given wavelength. For brevity, the wavelength dependence of the constants $A_{1}, \ldots, A_{n}$ has not been included.

At the beginning of an experiment, the total intensity at any wavelength is given by the sum of the individual contributions from the different fluorophores emitting at that wavelength. The fluorescence intensity under normal circumstances is linearly related to the quantum yield, the incident beam intensity, and the effective absorption cross section. These factors can be treated as a wavelength dependent constant of proportionality.

$$
I(\lambda)=\sum_{i=1}^{n} P_{i}(\lambda) A_{i}(\lambda)
$$


where $A_{i}$ and $P_{i}$ are the amount and proportionality constant of the $i^{\text {th }}$ flurophore. Since the $P_{i}$ values were assumed to be constant over the course of the experiment, they were dropped from further consideration with the product of $P_{i}$ and $A_{i}$ simply denoted by $A_{i}$. It should be noted that, if necessary, equation 12 can be adjusted to account for variable incident beam intensity, twophoton, and other non-linear effects. If there is no FRET occurring (e.g.: $\left.A_{n}+\ldots+A_{3}+A_{2}+A_{1}\right)$, then the fluorescence decay through photobleaching will fit to the exponential decay model:

$$
I(t, \lambda)=\sum_{i=1}^{n} A_{\mathrm{i}}(\lambda) e^{-k_{i} t}
$$

In this model, $A_{1}$ is assumed to be the most red-shifted fluorophore and the constants, $k_{i}$, are the wavelength independent rate constants for each component in the model.

If energy is being efficiently transferred, then, as an acceptor component decays, the corresponding donor will recover. If the rate of donor recovery is linked to the rate of decay of the acceptor this will appear as a component having the form of equations 9 and 10. For example, in an experiment with a single acceptor $\left(A_{1}\right)$ and a single donor $\left(A_{2}\right)$ such that $\left(A_{2} \rightarrow A_{1}\right)$, the growth and decay terms can be written as:

$$
I(t, \lambda)=A_{1}(\lambda) e^{-k_{1} t}+A_{2}(\lambda)\left(h_{12} e^{-k_{1} t}+h_{22} e^{-k_{2} t}\right)
$$

In contrast, the case $A_{2}+\left(A_{2} \rightarrow A_{1}\right)$, translates mathematically to: 
$I(t, \lambda)=A_{1}(\lambda) e^{-k_{1} t}+A_{21}(\lambda)\left(h_{12} e^{-k_{1} t}+h_{22} e^{-k_{2} t}\right)+A_{2}(\lambda) e^{-k_{2} t}$

15.

Here, $A_{21}$, and $A_{2}$ have been used to indicated the distinction between energy transfer and the parallel exponential decay.

In more complex systems of fluorophores, it is possible that many donors could transfer energy to the same acceptor. For example, $\left(A_{3} \rightarrow A_{1}\right)+\left(A_{2} \rightarrow A_{1}\right)$ might give a model with two growth and decay terms of the type given by equation 8 . The mathematical model would then be:

$I(t, \lambda)=A_{1}(\lambda) e^{-k_{1} t}+A_{21}(\lambda)\left(h_{12} e^{-k_{1} t}+h_{22} e^{-k_{2} t}\right)+A_{31}(\lambda)\left(h_{13} e^{-k_{1} t}+h_{33} e^{-k_{3} t}\right)$

16.

A three step process, $\left(A_{3} \rightarrow A_{2} \rightarrow A_{1}\right)$, would be represented by the following equation.

$I(t, \lambda)=A_{1}(\lambda) e^{-k_{1} t}+A_{2}(\lambda)\left(h_{12} e^{-k_{1} t}+h_{22} e^{-k_{2} t}\right)+A_{3}(\lambda)\left(h_{13} e^{-k_{1} t}+h_{23} e^{-k_{2} t}+h_{33} e^{-k_{3} t}\right)$ 17.

Finally, a fluorescent acceptor can bleach in a stepwise process. The first step might be an absorber that is no longer fluorescent. Non-fluorescent absorbers can still be efficient acceptors in systems undergoing energy transfer. In this case, this would appear as an sequential term with $A(\lambda)=0$

Statistical Tests for Parameter Inclusion and Model Selection. The myriad of kinetic models that can be used to describe a system of multiple fluorophores requires a method of testing which 
model best explains the measured data and whether additional terms in a model explain sufficient variance to justify their inclusion. In order to test whether additional parameters are justified, the F-test may be used to test the residual variances. This test may be applied in two ways: $a$ ) comparison of models with an equivalent number of parameters (e.g.: $\left(A_{2} \rightarrow A_{1}\right)$ vs. $\left.A_{2}+A_{1}\right)$; and b) comparison of models with a different number of parameters (e.g.: $A_{2}+\left(A_{2} \rightarrow A_{1}\right)$ vs. $\left(A_{2} \rightarrow\right.$ $\left.A_{1}\right)$ ). The latter test compares the sum of squared residuals for two models: a complete model with the greater number of parameters and a reduced model with fewer parameters. Since the sum of squared residuals decreases in a linear system when new parameters are added to the model, the question is whether this reduction is large enough to conclude that it is due to more than just an increase in the number of terms or to chance ${ }^{32}$. The deduction is based on the calculation of an $F$ statistic: $:$

$$
F_{v_{2}}^{v_{1}}=\frac{\frac{s s_{1}-s s_{2}}{k-g}}{\frac{s s_{2}}{n-k}}
$$

where $s s_{1}$ and $s s_{2}$, are the sum of squared residuals for the reduced and complete models, respectively; $k$ is the number of parameters for the complete model; $g$ is the number of parameters for the reduced model; $n$ is the number of data points; $k$ - $g$ corresponds to the number of degrees of freedom for the numerator, $v_{1}$; and $n-k$ corresponds to the number of degrees of

\footnotetext{
\$ The treatment given here is similar to one presented by Scheaffer and McClave ${ }^{32}$ The main difference between the two treatments is that the degrees of freedom for the denominator is given here as $n-k$ as opposed to $n-(k+1)$. The latter assumes that there is a constant term in the model that is not included in the value of $k$. See also Hanley et al. $^{34}$ for a description of related use of the F-test for model building.
} 
freedom for the denominator, $v_{2}$. The statistical parameters $s s_{1}$ and $s s_{2}$ are calculated according to the following equation:

$$
S s_{i}=\sum\left(I_{m, i}-I_{c, i}\right)
$$

where $I_{m, i}$ is the measured intensity and $I_{c, i}$ is the calculated intensity obtained from the fitting of the kinetic model.

The simplest model for any data set is a constant: $I(t, \lambda)=C(\lambda)$. The next simplest using the scheme presented here is $A_{1}$, the single exponential decay. Finally, the most reduced donor acceptor model is: $\left(A_{2} \rightarrow A_{1}\right)$. The hypotheses adopted for this statistical comparison are as follows:

$H_{0}=$ The additional parameters do not explain sufficient variance to justify their inclusion. $H_{a}=$ The inclusion of the additional parameters is justified.

$H_{0}$ is accepted when the computed $F$ statistic is less than the critical value for an $F$-distribution with $v_{1}$ and $v_{2}$ degrees of freedom. However, if the additional parameters contribute significantly to the model, the $F$ statistic would be larger than the critical $F$ and $H_{0}$ is rejected.

If the two kinetic models that are being compared have an equivalent number of parameters then equation 18 cannot be used to calculate the $F$-statistic. In this instance, the $F$-statistic is the ratio of the squared residuals: 


$$
F_{v_{2}}^{v_{1}}=\frac{S s_{1}}{S S_{2}}
$$

where $s s_{1}>s s_{2}$, so that $F_{\text {calculated }}>1$ and $v_{1}=v_{2}$. In this instance, the following hypotheses are adopted:

$H_{0}=$ The two models have an "equal” variance.

$H_{a}=$ One model explains significantly more variance than the other.

$H_{0}$ is accepted when the computed $F$ statistic is less than the critical value for an $F$ distribution with $v_{1}$ and $v_{2}$ degrees of freedom. However, if one model explains significantly more variance relative to the other model then $H_{0}$ is rejected when the calculated $F$ exceeds the critical value for the desired level of significance.

Estimating Errors in Fitted Parameters. The error in a coefficient obtained through least squares minimization may be estimated by numerical differentiation ${ }^{33}$. The standard deviation, $s_{i}$, of the $i^{\text {th }}$ coefficient, $a_{i}$, is: ${ }^{\S}$

$$
s_{i}=\sqrt{\mathbf{P}_{i, i}^{-1} *\left(\frac{s s_{\text {resid }}}{n-k}\right)}
$$

\footnotetext{
$\S$ The treatment here is substantially based on Billo ${ }^{33}$. This reference should be consulted for further details of the method.
} 
In equation 20, $\mathbf{P}_{i, i}^{-1}$ is the $\mathrm{i}^{\text {th }}$ diagonal element of the inverse of the matrix $\mathbf{P}$, ss $_{\text {resid }}$ is the residual variance for the model being evaluated, $n$ is the number of data points and $k$ is as in equation 18 . The elements of $\mathbf{P}$ are given by:

$$
\mathbf{P}_{i, j}=\sum_{n=1}^{N} \frac{\delta I}{\delta a_{i}} \frac{\delta I}{\delta a_{j}}
$$

The terms $\frac{\delta I}{\delta a_{i}}$ and $\frac{\delta I}{\delta a_{j}}$ are the partial derivatives of the function modeling the intensity decay, $I$, being fit with respect to the $i^{\text {th }}$ and $j^{\text {th }}$ coefficients. Numerically, these partial derivatives can be approximated as $\frac{\Delta I}{\Delta a_{i}}$ by making small changes in a coefficient and computing the change in $I$. In this fashion, the elements of $\mathbf{P}$ are computed. $\mathbf{P}$ is then inverted and the errors in the coefficients estimated.

\section{Experimental}

Instrumentation. The spectrally resolved photobleaching system was attached as an accessory to an inverted fluorescence microscope (E-300 Quantum; Nikon, New York, NY). Images were collected using a CCD camera (SensiCam Long Exposure; PCO Computer Optics GmbH, Kelheim, Germany). The camera was attached to the microscope via an imaging spectrograph (PARISS; Lightform Inc., Hillsborough, NJ) with $100 \mu \mathrm{m}$ slits. The imaging spectrograph was wavelength-calibrated relative to the $\mathrm{Hg}$ and $\mathrm{Ne}$ lines in a $\mathrm{Hg}$ pen lamp (Spectroline 11SC-1; Spectronics Corporation, Westbury, NY). Illumination was provided by a $\mathrm{Hg}$ arc lamp with a $365 \mathrm{~nm}$ excitation filter. Emission was through a $400 \mathrm{~nm}$ dichroic mirror and a $435 \mathrm{~nm}$ long pass 
filter. $^{* *}$ Photobleaching data for the fluorescent microspheres was acquired under computer control. A series of images were collected at regular time intervals during the illumination of the fluorescent microspheres with the $365 \mathrm{~nm}$ line of the $\mathrm{Hg}$ arc lamp.

Fluorescent microspheres designed to exhibit energy transfer (Models T-8869; Molecular Probes Inc., Oregon, USA) were diluted with distilled water in a micro-centrifuge tube. The T-8869 beads consisted of a system of 6 BODIPY ${ }^{\circledR}$ dyes: 4,4-difluoro-1,3-dipropyl-4-bora-3a,4a-diaza-sindacene; 4,4-difluoro-1,3-dipheny-5,7-dipropyl-4-bora-3a,4a-diaza-s-indacene; 4,4-difluoro1,3,5,7-tetraphenyl-4-bora-3a,4a-diaza-s-indacene; ４,4-difluoro-1,3-diphenyl.5.(2-pyrroly)-4bora-3a,4a-diaza-s-indacene; 4,4-difluoro(1-((3-(4methyloxyphenyl)-2H-isoinol-1-yl)methylene)3-(4-methoxyphenyl)1H-isoindolato- $\left.\mathrm{N}^{1}, \mathrm{~N}^{2}\right)$ boron; $\quad$ difluoro(5-methoxy-1-((5-methoxy-3-(4methoxyphenyl)-2H-isoindol-1-yl)methelene)-3-(4methoxyphenyl)-1H-isoindolato$\mathrm{N}^{1}, \mathrm{~N}^{2}$ )boron). ${ }^{30}$ A few drops of the dilute solution were placed in the centre of a glass slide and allowed to dry. A cover slip was then affixed over the sample. The sample was focused upon using a $40 \times$ objective (ELWD, NA=0.45, $\infty / 0-2, \mathrm{WD}=7.4$; Nikon, New York, NY).

Data Analysis. The kinetic models were least-squares fitted to a given fluorescence decay using the Solver tool in Microsoft ${ }^{\circledR}$ Office Excel. This analysis tool allows for minimization of the differences between the experimental intensity values and the calculated values using the applied kinetic model. Unless otherwise noted, the following constraints were placed on the fitted parameters: 1) All parameters were assumed to be non-negative; 2) Dyes were not allowed to emit more than $50 \mathrm{~nm}$ to the blue side of the emission maximum; 3) Dyes were not allowed to emit more than $100 \mathrm{~nm}$ to the red side of the emission maximum; 4) The emission associated

\footnotetext{
** This combination of filters was found to have low transmission above $\sim 700 \mathrm{~nm}$.
} 
with a particular rate constant was required to be smoothly increasing or decreasing (e.g.: a spectrum that reached 0 was not allowed to have further fluorescence on either the blue or red side of the emission maximum). Minimized values for the sum of squared residuals were obtained for the fitting of each multi-exponential function being considered in this study.

\section{Results and Discussion}

Spectral characteristics of the T-8869 microspheres. The spectra of the T-8869 fluorescent microspheres obtained during photobleaching (Figure 2) were initially dominated by a maximum at $690 \mathrm{~nm}$. As bleaching progressed, there was recovery of multiple donor fluorescence intensities in the red-green region of the spectrum with maxima appearing at approximately 675 $\mathrm{nm}, 635 \mathrm{~nm}, 600 \mathrm{~nm}, 560$, and $515 \mathrm{~nm}$ (expected: $678 \mathrm{~nm}, 635 \mathrm{~nm}, 592 \mathrm{~nm}, 560 \mathrm{~nm}$, and 515 $\mathrm{nm}$, respectively ${ }^{30}$ ). With the exception of the maximum observed at $690 \mathrm{~nm}$, all were in reasonable agreement with the reported emission maxima of the component dyes. The observed differences are attributable to the observation of the dyes in a mixture in which the spectra are heavily overlapped rather than in isolation. The exception at 690 (rather than 720) is attributable to blocking of the observed far red fluorescence by microscope components and reduced CCD camera quantum efficiency in the red portion of the spectrum.

Photobleaching of the T-8869 microspheres. Bleaching of the microspheres occurred over $\sim 60$ 80 minutes. After this time, the fluorescence intensity across the entire spectrum remained nearly unchanged except for a slow time scale $<0.03 \mathrm{~min}^{-1}$ ) bleaching process. During bleaching, growth and subsequent decay were most pronounced for the region between $550 \mathrm{~nm}$ and $650 \mathrm{~nm}$. Maximal growth (Figure 3) appeared after 10-20 minutes of bleaching depending on the wavelength of observation. 
Models constrained only to non-negativity were initially fit to the time course of photobleaching at six wavelengths with subtraction of a background based on the intensity observed at the end of bleaching (Table 1). Models consisting of exponential decay (models 2 and 3 in Table 1) were significantly better than a constant model. However, subtraction of the background will in general lower the significance of the constant terms and the exponential decay models gave poor fits. In particular, none of the exponential decay models exhibited the initial growth in fluorescence intensity seen in the data (Figure 3). In contrast, the simplest model including growth and decay based on the Bateman equations gave a fit explaining $>98 \%$ of the total variance. Of interest in the significance test comparing models 3 and 4 in Table 1 using equation 17 is that the computed $F$ evaluates to a negative number. This indicates that the reduced model explains more variance than the full model. Since these models are not linear systems, the common belief that an expression having more parameters will always fit a data set better than one with fewer parameters does not hold. Based on the results of these initial fittings, subsequent models consisting of solely decay terms were rejected and background was not subtracted.

Observation of the coefficients obtained from fits constrained only by non-negativity indicated the presence of intensity across the spectrum at the nominal beginning of the experiment. This was interpreted as photobleaching during the time required to setup the experiment and focus the specimen. Refinement of the time at which the experiment began was therefore included in later models.

Constrained fits to data from the T-8869 microspheres. A series of 9 global models consisting of between 1 and 7 photobleachable components were fitted to the time-course of photobleaching 
from a set of nine wavelengths $(713 \mathrm{~nm}, 690 \mathrm{~nm}, 674 \mathrm{~nm}, 650 \mathrm{~nm}, 641 \mathrm{~nm}, 610 \mathrm{~nm}, 588 \mathrm{~nm}, 562$ $\mathrm{nm}$, and $515 \mathrm{~nm}$ ). The models consisted of solutions to the Bateman equations (equations 8-10) and mixed models consisting of multi-step linked decay (equations 8-10) operating in parallel with simple exponential decay (equation 8) in the presence of an offset. The data-to-parameter ratio was in all cases 6.3 or greater. In the event that a parameter was found to be zero during the minimization process it was dropped from the model's parameter count. As expected, there was an increase in the fraction of the total variance explained as the number of parameters in the models increased (Figure 4). More importantly, the data justified the inclusion of parameters defining models up to Model 8 (Table 2). Fits to the constrained functions resulted in a good fit to the data and a reasonable representation of the expected behavior of BODIPY dyes (Figure 5), with the maxima lining up well with those observed in the emission spectra of the beads (Figure 1). The rate constants associated with the bleaching of the 6 fluorophores in the beads all fell within a similar range (Table 3). The best model fitting the data explained $>99.99 \%$ of the total variance with a data-to-parameter ratio of $>6.3$. This model included a time offset of $2.71 \pm 0.08$ min. Each addition of parameter sets while proceeding from Models 0-8 (Table 2) was justified and highly significant $(\mathrm{p}<0.001)$.

The degree to which the series of models fit the data suggests that there is little reason to invoke more complex mechanisms. It also generally supports Roberts et al. ${ }^{30}$ who found efficient energy transfer in these microspheres. Their results indicate $\sim 95 \%$ energy transfer efficiency to the terminal acceptor when excited at $365 \mathrm{~nm}$. If this is assumed to be the result of 5 successive energy transfer steps, then each step must be over $99 \%$ efficient. Model 8 suggests that the extent to which $100 \%$ energy transfer is not observed is likely due to the absence of terminal acceptors. 
The rapid energy transfer to a terminal acceptor is further supported by the known stoichiometry of the dyes in the beads, 12:1.8:1.7:1.3:1.0:1.1, with the species in highest concentration being the primary donor. Under these conditions, excitation energy most likely undergoes rapid energy migration via homo-transfer followed by hetero-transfer to the successive terminal acceptors.

\section{Conclusions}

This study demonstrates that spectroscopic photobleaching can be successfully used to elucidate mechanisms of FRET in complex mixtures of fluorophores. The model multi-fluorophore system, which was designed to exhibit FRET, provided the framework for the development of this analysis technique giving a theoretical basis for interpreting experimental data obtained through the photobleaching of a multi-fluorophore system. The current study demonstrates that very complex mechanisms of FRET can be tested, a statistically rigorous mechanism selected, and appropriate errors assigned. The T-8869 fluorescent microspheres exhibited a sequential mechanism of energy transfer where each terminal acceptor is sequentially bleached. Having reached this conclusion, a few cautionary notes need to be included. i) All the caveats which apply to studies of chemical reaction mechanisms using kinetic data apply here. Specifically, one does not prove a mechanism. Rather, mechanisms which are not supported by the data are rejected. Because of the complexity of multi-fluorophore systems, the models tested here should not be considered exhaustive. Thus, the mechanistic conclusions should be considered as only the best fit to the models based on the available data. This model can only be considered definitive until a better model is identified. ii) The strategy of model building and testing is similar to that used in other areas of science, perhaps most notably crystallography. In those fields, experience has shown that caution should be exercised when interpreting results. They should always be checked for reasonableness using any other available knowledge of the system and, when 
necessary, verified with additional experiments. iii) Fitting the Bateman equations to this data or similar data sets relies on the assumption of fast energy transfer. This means that there is only one pool of fluorophores that bleaches, those with no adjacent acceptor. We are currently investigating the behavior of systems of fluorophores in which this assumption does not necessarily hold. iv) The sequential mechanism of energy transfer should not be interpreted as meaning that it is obligatory that each time energy transfer occurs all the intermediate fluorophores must be involved. Rather, it means sequential production of a terminal acceptor. The five sequential energy transfer steps refer to transfer to sequentially produced terminal acceptors. The assumption of fast energy transfer means that direct transfer from $A_{6}$ to $A_{1}$ is indistinguishable from stepwise transfer from $A_{6}$ to $A_{5}$ to $A_{4}$ to $A_{3}$ to $A_{2}$ to $A_{1}$.

One limitation of the fluorescent microsphere system is that the most blue-shifted fluorophore appears to be the least robust with respect to photobleaching (Table 3). Kinetically, the best approach to engineering a photobleachable system of dyes is to select fluorophores such that they are increasingly photo-robust as their emission moves to the blue portion of the spectrum. For example, when investigating a sequential system undergoing energy transfer (e.g. model 8), a good approach is to select progressively more robust fluorophores as the fluorophore index increases (e.g.: for model 8, $k_{6}>k_{5}>k_{4}>k_{3}>k_{2}>k_{1}$ ). This will assure that a good build-up of donors will occur as the acceptors bleach.

This analysis technique is not limited to ideal systems but could be applied to the analysis of unknown biological systems in a variety of ways. For example, a set of fluorescent probes consisting of multiple donors representing putative binding partners for a single acceptor could 
be applied to a preparation to determine if one or perhaps many undergo FRET to the acceptor. Other examples consisting of such strategies as multiple donor-acceptor pairs or a single donor for multiple acceptors can be envisioned. However, in the case that the rate of energy transfer is not large, as was assumed here, modification of the models will be necessary to account for bleaching free donors and donors in the $\mathrm{D}^{*} \mathrm{~A}$ state. Another modification that might be introduced is the inclusion of models in which a distribution of donor-acceptor distances is present. As the technique of spectrally resolved pbFRET develops, it should be possible to treat more complex systems such as protein-protein interactions within marine organisms in order to better understand the photo-protective mechanisms of hard or reef building corals or signal transduction pathways probed with multiple fluorophores.

\section{Acknowledgements}

The authors thank Drs. Dmitry Cherny and Rainer Heintzmann at the Max Planck Institut für biophysikalische Chemie for helpful discussions during the preparation of this manuscript.

\section{References}

1. L. Stryer. Annual Review of Biochemistry 1978 47, 819.

2. T. Ha, T. Enderle, D. F. Ogletree, D. S. Chemla, P. R. Selvin and S. Weiss. Proceedings of the National Academy of Sciences of the United States of America 1996 93, 6264.

3. F. S. Wouters, P. J. Verveer and P. I. H. Bastiaens. Trends in Cell Biology 2001 11, 203.

4. F. J. Kleima, E. Hofmann, B. Gobets, I. H. M. van Stokkum, R. van Grondelle, K. Diederichs and H. van Amerongen. Biophysical Journal 2000 78, 344.

5. A. K. Kenworthy, N. Petranova and M. Edidin. Molecular Biology of the Cell 2000 11, 1645.

6. M. C. Overton and K. J. Blumer. Current Biology 2000 10, 341.

7. K. A. Eidne, K. M. Kroeger and A. C. Hanyaloglu. Trends in Endocrinology and Metabolism 2002 13, 415.

8. A. Kinoshita, H. Fukumoto, T. Shah, C. M. Whelan, M. C. Irizarry and B. T. Hyman. Journal of Cell Science 2003 116, 3339.

9. P. R. Selvin. Nature Structural Biology 2000 7, 730.

10. E. A. Jares-Erijman and T. M. Jovin. Nature Biotechnology 2003 21, 1387.

11. J. V. Goldstone, R. D. Vecchio, N. V. Blough and B. M. Voelker. Photochemistry and Photobiology 2004 80, 52. 
12. R. D. Vecchio and N. V. Blough. Environmental Science and Technology 2004 38, 3885.

13. R. D. Vecchio and N. V. Blough. Marine Chemistry 2002 78, 231.

14. L. L. Song, E. J. Hennink, I. T. Young and H. J. Tanke. Biophysical Journal 1995 68, 2588.

15. L. L. Song, R. P. van Gijlswijk, I. T. Young and H. J. Tanke. Cytometry 1997 27, 213.

16. L. L. Song, C. Varma, J. W. Verhoeven and H. J. Tanke. Biophysical Journal 1996 70, 2959.

17. L. Song. Ph. D. Thesis, Photobleaching Kinetics of Fluorescein in Quantitative Fluorescence Microscopy. University of Leiden, 1996.

18. P. S. Dittrich and P. Schwille. Applied Physics B-Lasers and Optics 2001 73, 829.

19. J. Lippincott-Schwartz, E. Snapp and A. Kenworthy. Nature Reviews Molecular Cell Biology 2001 2, 444.

20. J. White and E. Stelzer. Trends in Cell Biology 1999 9, 61.

21. P. I. H. Bastiaens and R. Pepperkok. Trends in Biochemical Sciences 2000 25, 631.

22. J. P. Szollosi, P. Nagy, Z. Sebestyen, S. Damjanovitch, J. W. Park and L. Matyus. Reviews in Molecular Biotechnology 2002 82, 251.

23. R. M. Young, J. K. Arnette, D. A. Roess and B. G. Barisas. Biophysical Journal 1994 67, 881.

24. U. Kubitscheck, R. Schweitzer-Stenner, D. J. Arndt-Jovin and T. M. Jovin. Biophysical Journal 1993 64, 110.

25. R. C. Patel, D. C. Lange and Y. C. Patel. Methods 2002 27, 340.

26. G. Szabo, P. S. Pine, J. L. Weaver, M. Kasari and A. Aszalos. Biophysical Journal 1992 61, 661.

27. M. A. Bopp, Y. W. Jia, L. Q. Li, R. J. Cogdell and R. M. Hochstrasser. Proceedings of the National Academy of Sciences of the United States of America 1997 94, 10630.

28. D. M. Benson, J. Bryan, A. L. Plant, A. M. Gotto and L. C. Smith. Journal of Cell Biology 1985 100, 1309.

29. T. W. J. Gadella Jr. and T. M. Jovin. Bioimaging 1997 5, 19.

30. D. V. Roberts, B. P. Wittmershaus, Y. Z. Zhang, S. Swan and M. P. Klinosky. Journal of Luminescence 1998 79, 225.

31. R. D. Evans. The Atomic Nucleus (McGraw-Hill, 1955).

32. R. L. Scheaffer and J. T. McClave. Probability and Statistics for Engineers (Duxbury Press, 1995).

33. E. J. Billo. Excel for Chemists: A Comprehensive Guide (Wiley-VCH, 2001).

34. Q. S. Hanley, J. W. Campbell and M. B. Denton. Journal of Synchrotron Radiation 19974 , 214. 

Table 1 Initial fits using non-negativity constraints

\begin{tabular}{|c|l|c|c|c|}
\hline $\begin{array}{c}\text { Model } \\
\text { number }\end{array}$ & Model & $\begin{array}{c}\text { Number } \\
\text { of } \\
\text { parameters }\end{array}$ & $\begin{array}{c}\text { Residual } \\
\text { variance }\end{array}$ & $\begin{array}{c}\text { Fraction } \\
\text { explained }\end{array}$ \\
\hline 0 & None & 0 & 11723459 & 0.0000 \\
\hline 1 & $C$ & 6 & 10249370 & 0.1257 \\
\hline 2 & $A_{1}$ & 7 & 2745871 & 0.7658 \\
\hline 3 & $A_{1}+A_{2}+A_{3}+A_{4}+A_{5}+A_{6}$ & 15 & 1698235 & 0.8551 \\
\hline 4 & $\left(A_{2} \rightarrow A_{1}\right)$ & 13 & 178029 & 0.9848 \\
\hline
\end{tabular}

Total number of data points $=174$. This set of fits was done using only non-negativity constraints. Note that model 4 fits the data better with fewer parameters than model 3. See text for details.

Table 2 Spectrally constrained fits

\begin{tabular}{|c|l|c|c|c|}
\hline $\begin{array}{c}\text { Model } \\
\text { number }\end{array}$ & \multicolumn{1}{|c|}{ Model } & $\begin{array}{c}\text { Number } \\
\text { of } \\
\text { parameters }\end{array}$ & $\begin{array}{c}\text { Residual } \\
\text { variance }\end{array}$ & $\begin{array}{c}\text { Fraction } \\
\text { explained }\end{array}$ \\
\hline 0 & None & 0 & 27225703 & 0.00000 \\
\hline 1 & $C$ & 9 & 18381910 & 0.32483 \\
\hline 2 & $A_{1}+C$ & 19 & 3811284 & 0.86001 \\
\hline 3 & $A_{2}+A_{1}+C$ & 21 & 2432264 & 0.91066 \\
\hline 4 & $A_{3}+\left(A_{2} \rightarrow A_{1}\right)+C$ & 27 & 1810268 & 0.93350 \\
\hline 5 & $A_{4}+\left(A_{3} \rightarrow A_{2} \rightarrow A_{1}\right)+C$ & 34 & 370409 & 0.98639 \\
\hline 6 & $A_{5}+\left(A_{4} \rightarrow A_{3} \rightarrow A_{2} \rightarrow A_{1}\right)+C$ & 39 & 38863 & 0.99857 \\
\hline 7 & $A_{6}+\left(A_{5} \rightarrow A_{4} \rightarrow A_{3} \rightarrow A_{2} \rightarrow A_{1}\right)+C$ & 40 & 7041 & 0.99974 \\
\hline 8 & $A_{7}+\left(A_{6} \rightarrow A_{5} \rightarrow A_{4} \rightarrow A_{3} \rightarrow A_{2} \rightarrow A_{1}\right)$ & 43 & 2232 & 0.99991 \\
\hline & $+C$ & & & \\
\hline
\end{tabular}

Total number of data points $=279$. 
Table 3 Rate constants associated with Model 8

\begin{tabular}{|c|c|}
\hline Rate constant & $\left(\mathrm{min}^{-1}\right)$ \\
\hline$k_{1}$ & $0.334 \pm 0.053$ \\
\hline$k_{2}$ & $0.143 \pm 0.004$ \\
\hline$k_{3}$ & $0.244 \pm 0.028$ \\
\hline$k_{4}$ & $0.108 \pm 0.004$ \\
\hline$k_{5}$ & $0.233 \pm 0.011$ \\
\hline$k_{6}$ & $0.466 \pm 0.113$ \\
\hline$k_{7}$ & $0.0245 \pm 0.0006$ \\
\hline
\end{tabular}

Constants $k_{1}$ to $k_{6}$ are associated with the dyes in the microspheres. Errors assigned using equations 21 and 22 .

\section{Figure Captions:}

Figure 1 In this diagram, the donor, D, may exist in two environments: i) as part of a donoracceptor complex (DA) or ii) as a free donor molecule (D). Donor molecules are excited via a

Table 3 Rate constants associated with Model 8

\begin{tabular}{|c|c|}
\hline Rate constant & $\left(\mathrm{min}^{-1}\right)$ \\
\hline$k_{1}$ & $0.334 \pm 0.053$ \\
\hline$k_{2}$ & $0.143 \pm 0.004$ \\
\hline$k_{3}$ & $0.244 \pm 0.028$ \\
\hline$k_{4}$ & $0.108 \pm 0.004$ \\
\hline$k_{5}$ & $0.233 \pm 0.011$ \\
\hline$k_{6}$ & $0.466 \pm 0.113$ \\
\hline$k_{7}$ & $0.0245 \pm 0.0006$ \\
\hline
\end{tabular}

first order intensity dependent process denoted by $I k_{e d}$ where $I$ is the intensity and $k_{e d}$ is the rate constant for donor excitation. This process is independent of whether the donor is paired with an acceptor or free. Free donor molecules are excited to $\mathrm{D}^{*}$ from which they may either deactivate at a rate governed by the rate constants for radiative $\left(k_{r d}\right)$ and non-radiative $\left(k_{n d}\right)$ deactivation or 
bleach according to the rate constant for free donor bleaching $\left(k_{b f d}\right)$. A donor-acceptor complex can be excited to $\mathrm{D}^{*} \mathrm{~A}$ or the acceptor can be excited directly producing DA* (according to the intensity, $I$, and the rate of acceptor excitation in the donor-acceptor complex, $k_{\text {eda }}$ ). Under saturating intensity levels, $\mathrm{D}^{*} \mathrm{~A}$ can undergo further excitation to produce doubly excited $\mathrm{D}^{*} \mathrm{~A}^{*}$. This latter process is included for completeness but will be neglected in the remainder of this paper as it is not favored under normal laboratory conditions. D*A may be converted to DA* via energy transfer at a rate denoted $k_{F R E T}$, or it can decay to the ground state via the radiative (fluorescence) or non-radiative (dark) processes. DA* can bleach producing free donor via a first order process with rate $k_{b a}$ or return to the ground state via the radiative and non-radiative pathways whose rate constants are indicated by $k_{r a}$ and $k_{n a}$, respectively. Finally, the donor molecule in $\mathrm{D}^{*} \mathrm{~A}$ can bleach via $k_{b d}$ to form B.

Figure 2 Spectra of the T-8869 microspheres during photo bleaching. Spectra shown are from the first 70 minutes of bleaching.

Figure 3 Time course of photobleaching observed at $560 \mathrm{~nm}$. At this wavelength, three processes can be clearly seen. i) Initial growth of fluorescence due to the photo-destruction of acceptors (020 minutes). ii) Relatively fast photo-destruction as the fluorophores present at this wavelength are bleached (30-80 minutes). iii) Slow residual photobleaching and offset (90-300 minutes).

Figure 4 Intermediate fits. Models fitted were: a) $C$ (9 parameters: 9 coefficients and no rate constants, fraction of total variance explained 0.3248$)$; b) $A_{1}+C$ (19 coefficients remained after fitting: 1 rate constant and 18 coefficients, fraction of total variance explained 0.8600$)$; c) $A_{3}+\left(A_{2}\right.$ $\left.\rightarrow A_{1}\right)+C$ (27 coefficients: 3 rate constants and 24 coefficients, fraction of total variance explained 
0.9353). d) $A_{6}+\left(A_{5} \rightarrow A_{4} \rightarrow A_{3} \rightarrow A_{2} \rightarrow A_{1}\right)+C$ (40 coefficients: 6 rate constants and 34 coefficients, fraction of total variance explained 0.9997). The important trend observed in going from panel a) to d) is the degree to which the model fits the position of the maxima in the time courses.

Figure 5 Final fit. The top panel shows the fits to the time course of photobleaching at 713, 690, $674,650,641,610,588,562$, and $515 \mathrm{~nm}$. The lower panel is a plot of the recovered spectra from the constrained analysis. Error bars are based on equations 22 and 23. This model explained > $99.99 \%$ of the total variance with a data-to-parameter ratio of $>6.3$. 


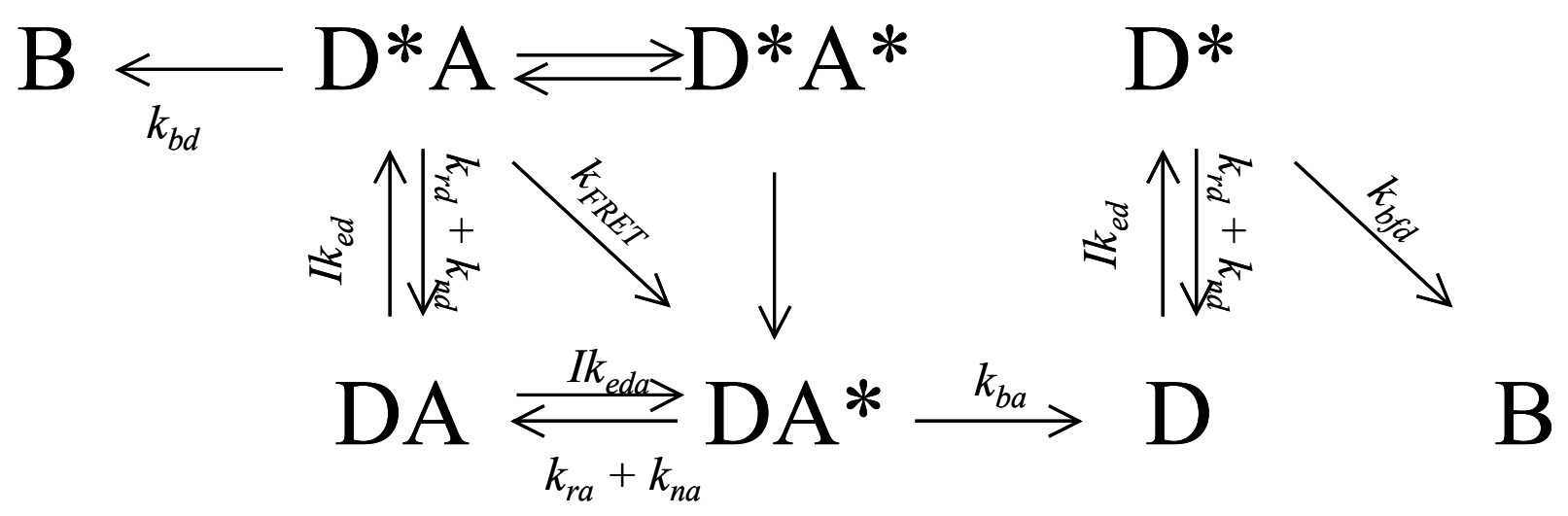




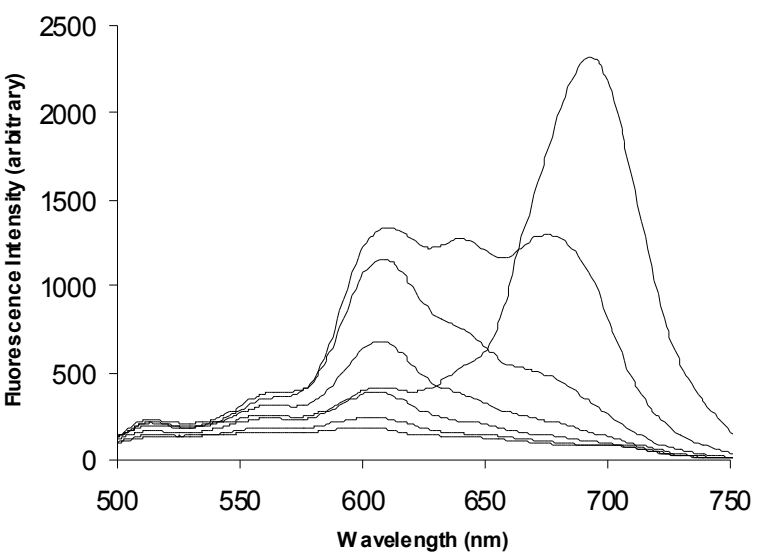




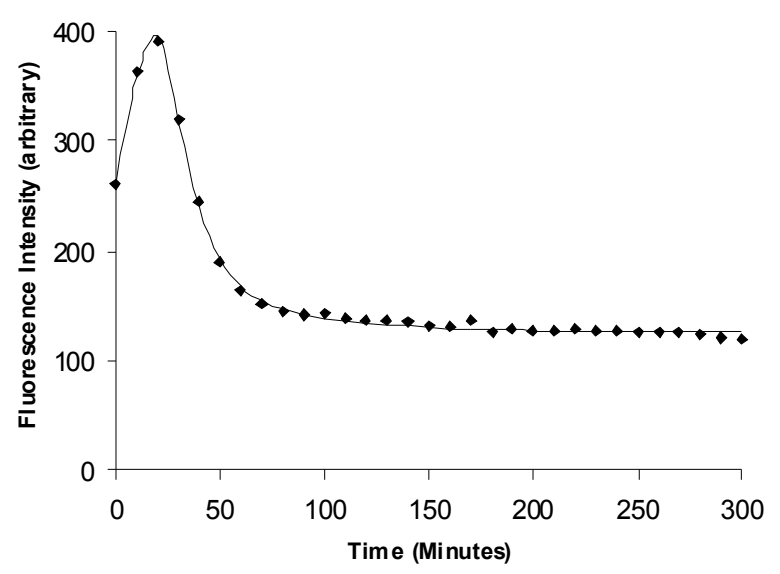



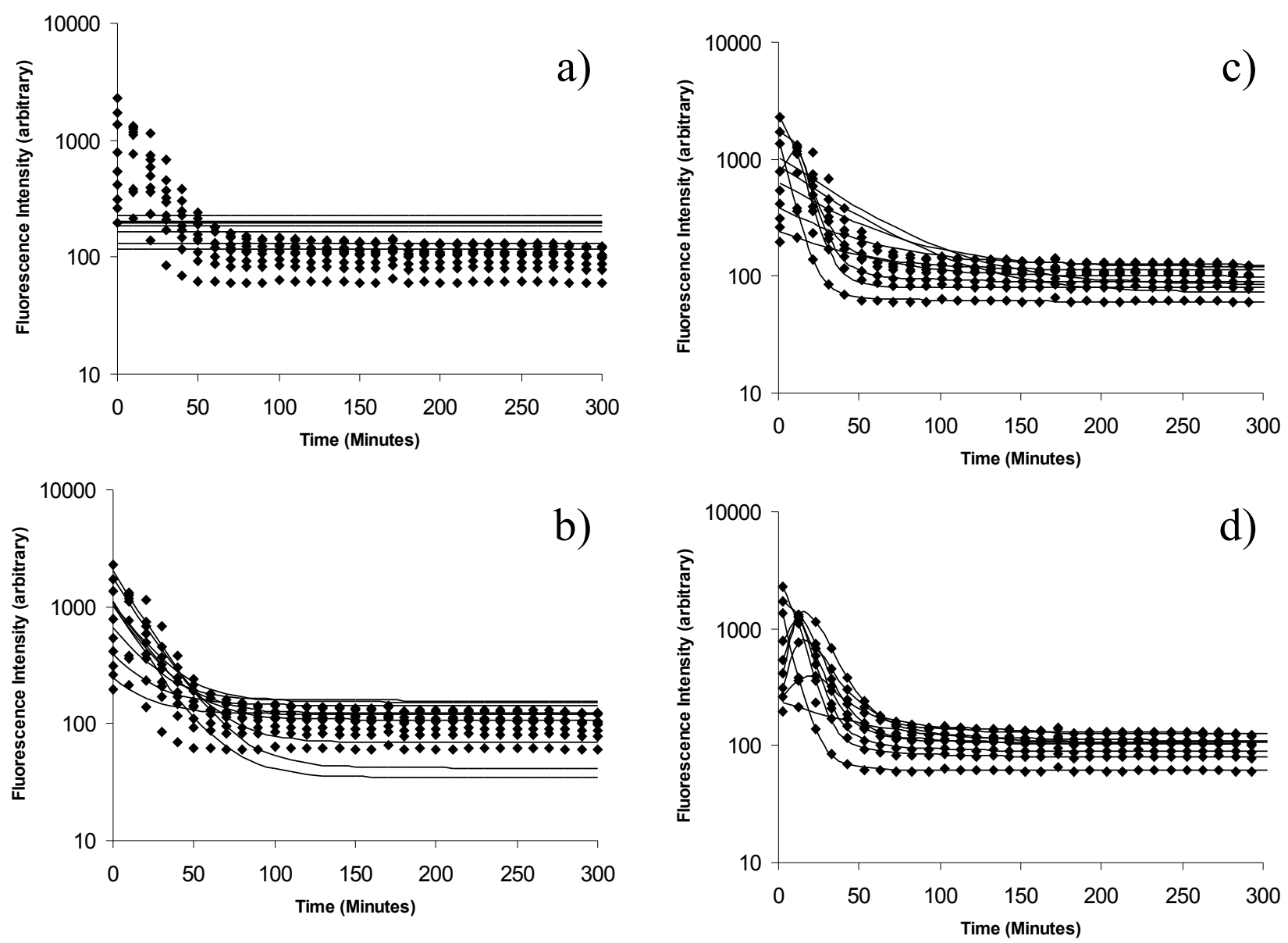

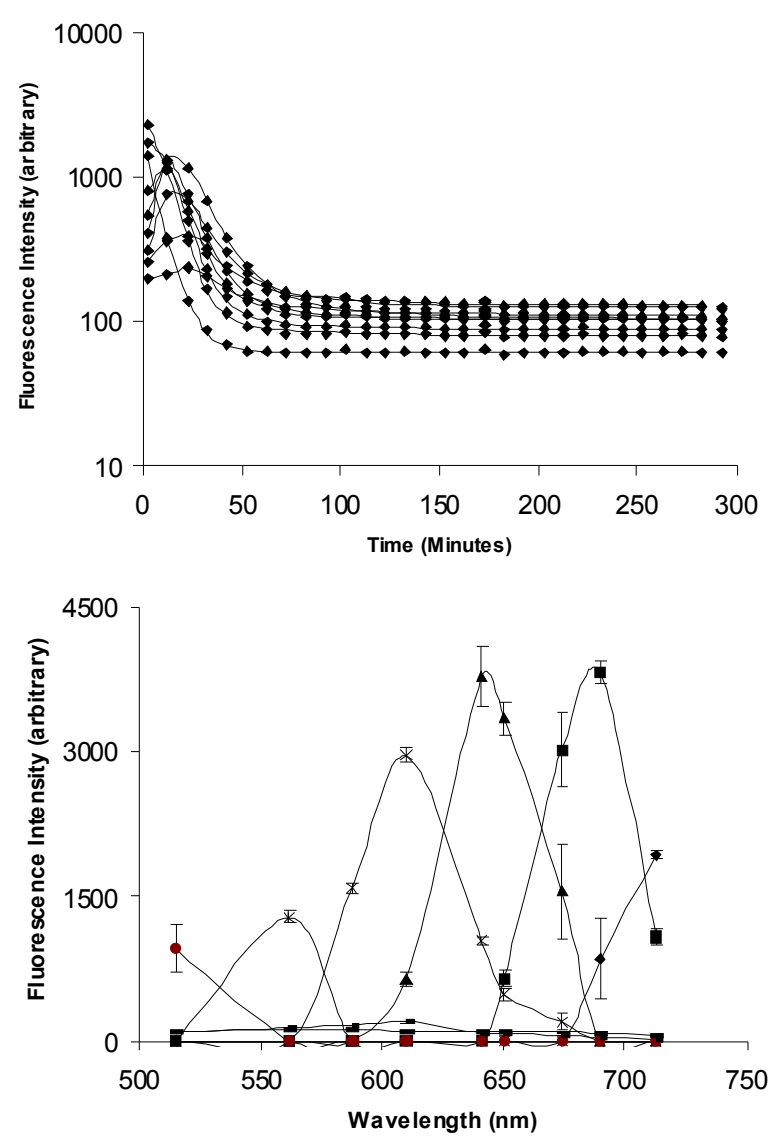